\title{
Effect of Drainage Parameters Change on Amount of Drain Discharge in Subsurface Drainage Systems
}

\author{
Mohammad Valipour \\ Department of Irrigation and Drainage Engineering, College of Abureyhan, University of Tehran, Pakdasht, \\ Tehran, Iran,
}

\begin{abstract}
One of the most important factors for appropriate performance of subsurface drainage systems is having adequate discharge for drains. For this purpose, knowledge about effect of drainage parameters change on drain discharge is essential in subsurface drainage systems. In this article, using change all of the drainage parameters by EnDrain software, changes of drains discharge has been investigated in subsurface drainage systems. The most amount of change in drain discharge for one percent increase or decrease in each of drainage parameters was owned by depth of water level in drain below soil surface equal to 3.0\%. Also about maximum and minimum of obtained drain discharge for drainage parameters change has been discussed.
\end{abstract}

Keywords: Drain discharge, subsurface drainage systems design, watertable control

\section{Introduction}

The subsurface drainage discharge is one of the most important indicators of the impact of the drainage systems on the water management. Many researches have been done about effect of drainage parameters and correct design of subsurface drainage, which some of them will be described in the following.

Rimidis and Dierickx (2003) evaluated subsurface drainage performance in Lithuania. Oosterbaan (1988) studied agricultural criteria for subsurface drainage. Endres et al. (2007) compared analytical model predictions and field measurements for pumping-induced vadose zone drainage and storage in an unconfined aquifer. The delayed drainage models predicted a relatively rapid dissipation of the undrained storage while the observed undrained storage exhibited little, if any, decay throughout the entire pumping test. Their results indicated that the water table boundary conditions used in these analytical models did not adequately replicate the mechanisms controlling the vadose zone behavior during a pumping test. Cooke et al. (2001) studied drainage equations for random and irregular tile drainage systems. The results predicted by the derived solution were found to be in close agreement with those obtained from the numerical simulations. Therefore, it was concluded that the proposed model holds well for situations of practical import and could be used in future work with large-scale hydrologic models. Howell et al. (2012) presented centrifuge modeling of prefabricated vertical drains for liquefaction remediation. Geng et al. (2012) presented analytical solutions for a single vertical drain with vacuum and time-dependents preloading in membrane and membraneless systems. The analytical solutions improved the accuracy of predicting the dissipation of pore water pressure and the associated settlement. Ghandeharioon et al. (2010) analyzed soil disturbance associated with mandrel-driven prefabricated vertical drains using an elliptical cavity expansion theory. Basu and Prezzi (2010) designed charts for vertical drains considering soil disturbance. The designed charts could also be used for conditions in which overlapping of disturbed zones occurs. Prasad et al. (2010) estimated unsaturated hydraulic parameters from infiltration and internal drainage experiments. Marinucci et al. (2010) evaluated the effectiveness of prefabricated vertical drains using full-scale in situ staged dynamic testing. Singh (2010) survived generalized analytical solutions for groundwater head in inclined aquifers in the presence of subsurface drains. Oosterbaan (2010) survived role of water harvesting and agricultural land development in spate irrigation in the NWFR of Pakistan. Coles (1968) investigated some notes on drainage design procedure. He showed that various formulae could be solved directly, but graphs have been included to simplify the solution of the different equations. Oosterbaan (1991) studied application of agricultural land drainage. Oosterbaan (1991) in another research discussed about effectiveness and social/environmental impacts of irrigation projects. Samani et al. (2004) studied flow to horizontal and slanted drains in anisotropic unconfined aquifers. Youngs (1986) discussed about water-table heights in drained anisotropic homogeneous soils. Barua and Tiwari (1995) presented theories of seepage into auger holes in homogeneous anisotropic soil. Singh et al. (1996) researched unsteady state drainage in a vertically heterogeneous soil. Endres et al. (2007) compared analytical model predictions and field measurements for pumping-induced vadose zone drainage and storage in an unconfined aquifer. The delayed drainage models predicted a relatively rapid dissipation of the undrained storage while the observed undrained storage exhibited little, if any, decay throughout the entire pumping test. Their results indicated that the water table boundary conditions used in these analytical models did not adequately replicate the mechanisms controlling the vadose zone behavior during a pumping test. Luan and Leng (2008) compared monotonic shear 
behaviors of granular soils under different drainage conditions. Ali (2011) studied drainage of agricultural lands as a comprehensive research. O'Kelly (2006) compared anisotropy of some soft soils. Gallichand (1994) presented numerical simulations of steady-state subsurface drainage with vertically decreasing hydraulic conductivity. The results presented could be used to estimate the error on watertable depth resulting from ignoring the vertical variations of hydraulic conductivity. Hunt (2005) discussed about flow to vertical and nonvertical wells in leaky aquifers. Choudhry et al. (1995) showed Physical and hydraulic properties of synthetic envelopes for subsurface drainage in Pakistan. Hanson and Ayars (2002) presented strategies for reducing subsurface drainage in irrigated agriculture through improved irrigation. Kannan (2008) Studied drawdown-drain discharge relationship and its application in design of Ccost effective subsurface drainage system in Mugogo Swamp, Busogo, Rwanda. Osiensky et al. (2000) evaluated drawdown curves derived from multiple well aquifer tests in heterogeneous environments. Moustafa (1998) survived time-dependent drainage from root zone and drainage coefficient under different irrigation management levels for subsurface drainage design in Egypt. O'Neill et al. (1989) presented agricultural subsurface drainage from potato fields in northwestern New Brunswick, Canada. Wahba and Christen (2006) modeled subsurface drainage for salt load management in southeastern Australia. Hornbuckle et al. (2005) managed controlled water table management as a strategy for reducing salt loads from subsurface drainage under perennial agriculture in semi-arid Australia. Results from the experiment showed that controlled drainage significantly reduced drainage volumes and salt loads compared to unmanaged systems. However, there were marked increases in soil salinity which will need to be carefully monitored and managed. Christen et al. (2001) designed subsurface drainage in irrigated areas of Australia, successfully. Castanheira and Santos (2009) presented a simple numerical analyses software for predicting water table height in subsurface drainage. The results obtained with the model agree well with Khirkam's and Hooghoudt analytical solution for the distribution of total head in ideal drains and for the total head calculations midway between drains. Burdon (1986) investigated hydrogeological aspects of agricultural drainage in Ireland, successfully. Ahmadi (1995) using a field approach estimated drainage coefficients in humid area. Brandyk et al. (1992) using a simple flow resistance model managed drainage/sub-irrigation systems. Wesseling (1964) compared the steady state drain spacing formulas of Hooghoudt and Kirkham in connection with design practice. Molen and Wesseling (1991) presented a solution in closed form and a series solution to replace the tables for the thickness of the equivalent layer in Hooghoudt's drain spacing formula. Wesseling (1964) studied The effect of using continually submerged drains on drain spacing. Singh et al. (1992) survived modified steady state drainage equations for transient conditions in subsurface drainage. Lovell and Youngs (1984) compared steady-state land-drainage equations. Of the drainage equations Houghoudt's equivalent depth equation, when used with the optimum drain radius given by the hodograph analysis for infinite soil depth, was the only one that gives results contained mainly within the known bounds that result from a consideration of the combination of equations. Youngs (1985) presented a simple drainage equation for predicting water-table drawdowns. This simple equation was useful in the analysis of falling water tables in drained lands. Singh et al. (1999) survived subsurface drainage of a three layered soil with slowly permeable top layer. The study showed that the watertable head gets influenced by the location of interface between the soil layers. French and O'Callaghan (1966) described a field-test of drain spacing equations for agricultural land. Wiskow and Ploeg (2003) calculated drain spacing for optimal rainstorm flood control. Hirekhan et al. (2007) showed application of WaSim to assess performance of a subsurface drainage system under semi-arid monsoon climate. It appeared that WaSim was a simple tool to evaluate the hydraulic performance of the subsurface drainage systems or to design a subsurface drainage system for semi-arid monsoon climates. Prasher et al. (1994) designed water table management systems in humid areas as economical. Nwa and Twocock (1969) discussed about drainage design theory and practice. Skagges et al. (2006) studied drainage design coefficients for eastern United States. Singh and O'Callaghan (1978) investigated non-steady drainage in a layered soil. Youngs (1986) determined the variation of hydraulic conductivity with depth in drained lands and the design of drainage installations. Gureghian and Youngs (1975) using finite-element method calculated steady-state watertable heights in drained soils. Youngs (1991) in other research said a note on the power-law land-drainage equation for deep soils. Valipour (2012) compared two types subsurface drainage system (horizontal and vertical) in anisotropic soils. He showed that changes of hydraulic conductivity had a significant effect on drain spacing.

Most previous studies focused on drainage spacing and neglected role of all drainage parameters in subsurface drainage systems. In this study, using change all of the drainage parameters by EnDrain software, changes of drains discharge has been investigated in subsurface drainage systems.

\section{Materials And Methods}

In this study simulated performance of subsurface drainage by using EnDrain software. The drain discharge calculations in this software were based on the Darcy and waterbalance (water balance, budget) or mass conservation equations. In this paper presented ten different scenarios for each of drainage parameters. For 
each scenarios amount of drain discharge changes obtained and compared. The eight drainage parameters witch survived effect of their changes on drain discharge were depth watertable midway between drains $(\mathrm{Dm})$, bottom depth of layer below soil surface $(D)$, depth of water level in drain below soil surface $(D w)$, depth of drain bottom below soil surface $(D d)$, entrance resistance at the drain $(E)$, maximum width of water body in the drain $(W)$, hydraulic permeability $(K)$, and spacing between the parallel drains $(S)$. The amount of entrance resistance at the drain calculated as follows:

$E=H_{e} / Q^{*}$

Where $H_{e}$ is entrance head (m) and $Q^{*}$ is drain discharge ( $\mathrm{m}^{2} /$ day) which as follows:

$Q^{*}=R \times S$

Where $R$ is amount of recharge (m/day).

The initial data were $\mathrm{Dm}=1.0 \mathrm{~m}, \mathrm{D}=6.3 \mathrm{~m}, \mathrm{Dw}=1.5 \mathrm{~m}, \mathrm{Dd}=1.6 \mathrm{~m}, \mathrm{E}=0.5 \mathrm{day} / \mathrm{m}, \mathrm{W}=0.2 \mathrm{~m}, \mathrm{~K}=0.14 \mathrm{~m} / \mathrm{day}, \mathrm{S}=65$ $\mathrm{m}$ and have been highlighted in all of the tables in this paper. For these amounts, drain discharge calculated using EnDrain software equal to $0.0009 \mathrm{~m} /$ day.

\section{Results And Discussion}

Table 1 shows obtained results for change of depth watertable midway between drains.

Table 1. Obtained results for change of depth watertable midway between drains (Dm)

\begin{tabular}{|c|c|c|c|c|c|c|c|c|c|c|c|}
\hline $\mathrm{Dm}(\mathrm{m})$ & $\begin{array}{l}\mathrm{D} \\
(\mathrm{m})\end{array}$ & $\begin{array}{l}\text { Dw } \\
(\mathrm{m})\end{array}$ & $\begin{array}{l}\mathrm{Dd} \\
(\mathrm{m})\end{array}$ & $\begin{array}{c}\mathrm{E} \\
(\mathrm{day} / \mathrm{m})\end{array}$ & $\begin{array}{l}\mathrm{W} \\
(\mathrm{m})\end{array}$ & $\begin{array}{c}\mathrm{K} \\
\text { (m/day) }\end{array}$ & $\begin{array}{c}\mathrm{S} \\
(\mathrm{m})\end{array}$ & $\begin{array}{l}\text { Drain discharge } \\
\text { (m/day) }\end{array}$ & $\begin{array}{c}\Delta \mathrm{Q} / \mathrm{Q} \\
(\%)\end{array}$ & $\begin{array}{c}\Delta \mathrm{Dm} / \mathrm{Dm} \\
(\%)\end{array}$ & $\begin{array}{c}\text { Final } \\
\text { change } \\
(\%)\end{array}$ \\
\hline 0.1 & 6.3 & 1.5 & 1.6 & 0.5 & 0.2 & 0.14 & 65 & 0.0030 & 233 & 90 & 2.6 \\
\hline 0.2 & 6.3 & 1.5 & 1.6 & 0.5 & 0.2 & 0.14 & 65 & 0.0027 & 200 & 80 & 2.5 \\
\hline 0.4 & 6.3 & 1.5 & 1.6 & 0.5 & 0.2 & 0.14 & 65 & 0.0022 & 144 & 60 & 2.4 \\
\hline 0.6 & 6.3 & 1.5 & 1.6 & 0.5 & 0.2 & 0.14 & 65 & 0.0018 & 100 & 40 & 2.5 \\
\hline 0.8 & 6.3 & 1.5 & 1.6 & 0.5 & 0.2 & 0.14 & 65 & 0.0013 & 44 & 20 & 2.2 \\
\hline 1.0 & 6.3 & 1.5 & 1.6 & 0.5 & 0.2 & 0.14 & 65 & 0.0009 & 0 & 0 & 0.0 \\
\hline 1.1 & 6.3 & 1.5 & 1.6 & 0.5 & 0.2 & 0.14 & 65 & 0.0007 & 22 & 10 & 2.2 \\
\hline 1.2 & 6.3 & 1.5 & 1.6 & 0.5 & 0.2 & 0.14 & 65 & 0.0005 & 44 & 20 & 2.2 \\
\hline 1.3 & 6.3 & 1.5 & 1.6 & 0.5 & 0.2 & 0.14 & 65 & 0.0004 & 56 & 30 & 1.9 \\
\hline 1.4 & 6.3 & 1.5 & 1.6 & 0.5 & 0.2 & 0.14 & 65 & 0.0001 & 89 & 40 & 2.2 \\
\hline $\begin{array}{c}\text { Average } \\
(\%)\end{array}$ & & & & & & & & & 104 & 43 & 2.3 \\
\hline
\end{tabular}

According to the Table 1 maximum amount of drain discharge change into the initial discharge $(\Delta \mathrm{Q} / \mathrm{Q})$ was $233 \%$ and related to the 90 percent of decreasing depth watertable midway between drains into the initial $\mathrm{Dm}$ $(\Delta \mathrm{Dm} / \mathrm{Dm})$. The minimum of changes was $22 \%$ for $10 \%$ increasing of $\mathrm{Dm}$.

The amount of final change calculated by dividing $\Delta \mathrm{Q} / \mathrm{Q}$ on $\Delta \mathrm{Dm} / \mathrm{Dm}$ therefore amount of 2.3 in Table 1 indicates that as average for one percent decrease or increase in $D m$, amount of drain discharge is changed $2.3 \%$.

Table 2 shows obtained results for bottom depth of layer below soil surface.

Table 2. Obtained results for bottom depth of layer below soil surface (D)

\begin{tabular}{cccccccccccc}
\hline $\operatorname{Dm}(\mathrm{m})$ & $\begin{array}{c}\mathrm{D} \\
(\mathrm{m})\end{array}$ & $\begin{array}{c}\mathrm{Dw} \\
(\mathrm{m})\end{array}$ & $\begin{array}{c}\mathrm{Dd} \\
(\mathrm{m})\end{array}$ & $\begin{array}{c}\mathrm{E} \\
(\mathrm{day} / \mathrm{m})\end{array}$ & $\begin{array}{c}\mathrm{W} \\
(\mathrm{m})\end{array}$ & $\begin{array}{c}\mathrm{K} \\
(\mathrm{m} / \text { day })\end{array}$ & $\begin{array}{c}\mathrm{S} \\
(\mathrm{m})\end{array}$ & $\begin{array}{c}\text { Drain discharge } \\
(\mathrm{m} / \text { day })\end{array}$ & $\begin{array}{c}\Delta \mathrm{Q} / \mathrm{Q} \\
(\%)\end{array}$ & $\begin{array}{c}\Delta \mathrm{D} / \mathrm{D} \\
(\%)\end{array}$ & $\begin{array}{c}\text { Final } \\
\text { change }(\%)\end{array}$ \\
\hline 1.0 & 2.0 & 1.5 & 1.6 & 0.5 & 0.2 & 0.14 & 65 & 0.0002 & 78 & 68 & 1.1 \\
1.0 & 4.0 & 1.5 & 1.6 & 0.5 & 0.2 & 0.14 & 65 & 0.0006 & 33 & 37 & 0.9 \\
1.0 & 6.3 & 1.5 & 1.6 & 0.5 & 0.2 & 0.14 & 65 & 0.0009 & 0 & 0 & 0.0 \\
1.0 & 8.0 & 1.5 & 1.6 & 0.5 & 0.2 & 0.14 & 65 & 0.0011 & 22 & 27 & 0.8 \\
1.0 & 10.0 & 1.5 & 1.6 & 0.5 & 0.2 & 0.14 & 65 & 0.0012 & 33 & 59 & 0.6 \\
1.0 & 15.0 & 1.5 & 1.6 & 0.5 & 0.2 & 0.14 & 65 & 0.0014 & 56 & 138 & 0.0 \\
1.0 & 20.0 & 1.5 & 1.6 & 0.5 & 0.2 & 0.14 & 65 & 0.0015 & 67 & 217 & 0.3 \\
1.0 & 30.0 & 1.5 & 1.6 & 0.5 & 0.2 & 0.14 & 65 & 0.0015 & 67 & 376 & 0.2 \\
1.0 & 40.0 & 1.5 & 1.6 & 0.5 & 0.2 & 0.14 & 65 & 0.0015 & 67 & 535 & 0.1 \\
1.0 & 50.0 & 1.5 & 1.6 & 0.5 & 0.2 & 0.14 & 65 & 0.0015 & 67 & 694 & 0.1 \\
Average & & & & & & & & & 54 & 239 & 0.5 \\
$(\%)$ & & & & & & & & & 54 & \\
\hline
\end{tabular}


According to the Table 2 maximum amount of drain discharge change into the initial discharge $(\Delta \mathrm{Q} / \mathrm{Q}) \mathrm{was} 78 \%$ and related to the $68 \%$ decreasing bottom depth of layer below soil surface into the initial $D(\Delta \mathrm{D} / \mathrm{D})$. The minimum of changes was $22 \%$ for $27 \%$ increasing of $\mathrm{Dm}$. As average for one percent decrease or increase in $\mathrm{D}$, amount of drain discharge is changed $0.5 \%$.

Table 3 shows obtained results for depth of water level in drain below soil surface.

Table 3. Obtained results for depth of water level in drain below soil surface (Dw)

\begin{tabular}{|c|c|c|c|c|c|c|c|c|c|c|c|}
\hline $\operatorname{Dm}(m)$ & $\begin{array}{c}\mathrm{D} \\
(\mathrm{m})\end{array}$ & $\begin{array}{l}\mathrm{Dw} \\
(\mathrm{m})\end{array}$ & $\begin{array}{l}\mathrm{Dd} \\
(\mathrm{m})\end{array}$ & $\begin{array}{c}\mathrm{E} \\
(\mathrm{day} / \mathrm{m})\end{array}$ & $\begin{array}{l}\mathrm{W} \\
(\mathrm{m})\end{array}$ & $\begin{array}{c}\mathrm{K} \\
\text { (m/day) }\end{array}$ & $\begin{array}{c}\mathrm{S} \\
(\mathrm{m})\end{array}$ & $\begin{array}{c}\text { Drain discharge } \\
\text { (m/day) }\end{array}$ & $\begin{array}{c}\Delta \mathrm{Q} / \mathrm{Q} \\
(\%)\end{array}$ & $\begin{array}{c}\Delta \mathrm{Dw} / \mathrm{Dw} \\
(\%)\end{array}$ & $\begin{array}{c}\text { Final change } \\
(\%)\end{array}$ \\
\hline 1.0 & 6.3 & 1.05 & 1.6 & 0.5 & 0.2 & 0.14 & 65 & 0.0001 & 89 & 30 & 3.0 \\
\hline 1.0 & 6.3 & 1.10 & 1.6 & 0.5 & 0.2 & 0.14 & 65 & 0.0002 & 78 & 27 & 2.9 \\
\hline 1.0 & 6.3 & 1.15 & 1.6 & 0.5 & 0.2 & 0.14 & 65 & 0.0002 & 78 & 23 & 3.3 \\
\hline 1.0 & 6.3 & 1.20 & 1.6 & 0.5 & 0.2 & 0.14 & 65 & 0.0003 & 67 & 20 & 3.3 \\
\hline 1.0 & 6.3 & 1.25 & 1.6 & 0.5 & 0.2 & 0.14 & 65 & 0.0003 & 67 & 17 & 4.0 \\
\hline 1.0 & 6.3 & 1.30 & 1.6 & 0.5 & 0.2 & 0.14 & 65 & 0.0006 & 33 & 13 & 2.5 \\
\hline 1.0 & 6.3 & 1.40 & 1.6 & 0.5 & 0.2 & 0.14 & 65 & 0.0008 & 11 & 7 & 1.7 \\
\hline 1.0 & 6.3 & 1.45 & 1.6 & 0.5 & 0.2 & 0.14 & 65 & 0.0009 & 0 & 3 & 0.0 \\
\hline 1.0 & 6.3 & 1.50 & 1.6 & 0.5 & 0.2 & 0.14 & 65 & 0.0009 & 0 & 0 & 0.0 \\
\hline 1.0 & 6.3 & 1.55 & 1.6 & 0.5 & 0.2 & 0.14 & 65 & 0.0011 & 22 & 3 & 6.7 \\
\hline $\begin{array}{c}\text { Average } \\
(\%)\end{array}$ & & & & & & & & & 49 & 16 & 3.0 \\
\hline
\end{tabular}

According to the Table 3 maximum amount of drain discharge change into the initial discharge $(\Delta \mathrm{Q} / \mathrm{Q}) \mathrm{was} 89 \%$ and related to the $30 \%$ decreasing depth of water level in drain below soil surface into the initial $D w(\Delta \mathrm{Dw} / \mathrm{Dw})$. The minimum of changes was $0 \%$ for $3 \%$ decreasing of $D w$. As average for one percent decrease or increase in $D w$, amount of drain discharge is changed $3.0 \%$.

Table 4 shows obtained results for depth of drain bottom below soil surface.

Table 4. Obtained results for depth of drain bottom below soil surface (Dd)

\begin{tabular}{|c|c|c|c|c|c|c|c|c|c|c|c|}
\hline $\operatorname{Dm}(\mathrm{m})$ & $\begin{array}{c}\mathrm{D} \\
(\mathrm{m})\end{array}$ & $\begin{array}{l}\mathrm{Dw} \\
(\mathrm{m})\end{array}$ & $\begin{array}{l}\mathrm{Dd} \\
(\mathrm{m})\end{array}$ & $\begin{array}{c}E \\
(\text { day/m) }\end{array}$ & $\begin{array}{l}\mathrm{W} \\
(\mathrm{m})\end{array}$ & $\begin{array}{c}\mathrm{K} \\
\text { (m/day) }\end{array}$ & $\begin{array}{c}\mathrm{S} \\
(\mathrm{m})\end{array}$ & $\begin{array}{c}\text { Drain discharge } \\
\text { (m/day) }\end{array}$ & $\begin{array}{l}\Delta \mathrm{Q} / \mathrm{Q} \\
(\%)\end{array}$ & $\begin{array}{c}\Delta \mathrm{Dd} / \mathrm{Dd} \\
(\%)\end{array}$ & $\begin{array}{c}\text { Final change } \\
(\%)\end{array}$ \\
\hline 1.0 & 6.3 & 1.5 & 1.6 & 0.5 & 0.2 & 0.14 & 65 & 0.0009 & 0 & 0 & 0.0 \\
\hline 1.0 & 6.3 & 1.5 & 1.7 & 0.5 & 0.2 & 0.14 & 65 & 0.0010 & 11 & 6 & 1.8 \\
\hline 1.0 & 6.3 & 1.5 & 1.8 & 0.5 & 0.2 & 0.14 & 65 & 0.0010 & 11 & 13 & 0.9 \\
\hline 1.0 & 6.3 & 1.5 & 1.9 & 0.5 & 0.2 & 0.14 & 65 & 0.0010 & 11 & 19 & 0.6 \\
\hline 1.0 & 6.3 & 1.5 & 2.0 & 0.5 & 0.2 & 0.14 & 65 & 0.0011 & 22 & 25 & 0.9 \\
\hline 1.0 & 6.3 & 1.5 & 2.2 & 0.5 & 0.2 & 0.14 & 65 & 0.0011 & 22 & 38 & 0.0 \\
\hline 1.0 & 6.3 & 1.5 & 2.5 & 0.5 & 0.2 & 0.14 & 65 & 0.0011 & 22 & 56 & 0.4 \\
\hline 1.0 & 6.3 & 1.5 & 3.0 & 0.5 & 0.2 & 0.14 & 65 & 0.0012 & 33 & 88 & 0.4 \\
\hline 1.0 & 6.3 & 1.5 & 3.5 & 0.5 & 0.2 & 0.14 & 65 & 0.0012 & 33 & 119 & 0.0 \\
\hline 1.0 & 6.3 & 1.5 & 4.0 & 0.5 & 0.2 & 0.14 & 65 & 0.0013 & 44 & 150 & 0.3 \\
\hline $\begin{array}{c}\text { Average } \\
(\%)\end{array}$ & & & & & & & & & 23 & 57 & 0.6 \\
\hline
\end{tabular}

According to the Table 4 maximum amount of drain discharge change into the initial discharge $(\Delta \mathrm{Q} / \mathrm{Q}) \mathrm{was} 44 \%$ and related to the $150 \%$ increasing depth of drain bottom below soil surface into the initial $D d(\Delta \mathrm{Dd} / \mathrm{Dd})$. The minimum of changes was $11 \%$ for 6-19\% increasing of $D d$. As average for one percent decrease or increase in $D d$, amount of drain discharge is changed $0.6 \%$.

Table 5 shows obtained results for entrance resistance at the drain.

Table 5. Obtained results for entrance resistance at the drain (E)

\begin{tabular}{|c|c|c|c|c|c|c|c|c|c|c|c|}
\hline $\operatorname{Dm}(\mathrm{m})$ & $\begin{array}{c}\mathrm{D} \\
(\mathrm{m})\end{array}$ & $\begin{array}{l}\text { Dw } \\
(\mathrm{m})\end{array}$ & $\begin{array}{l}\mathrm{Dd} \\
(\mathrm{m})\end{array}$ & $\begin{array}{c}\mathrm{E} \\
(\mathrm{day} / \mathrm{m})\end{array}$ & $\begin{array}{l}\mathrm{W} \\
(\mathrm{m})\end{array}$ & $\begin{array}{c}\mathrm{K} \\
\text { (m/day) }\end{array}$ & $\begin{array}{c}\mathrm{S} \\
(\mathrm{m})\end{array}$ & $\begin{array}{c}\text { Drain discharge } \\
(\mathrm{m} / \text { day })\end{array}$ & $\begin{array}{c}\Delta \mathrm{Q} / \mathrm{Q} \\
(\%)\end{array}$ & $\Delta \mathrm{E} / \mathrm{E}(\%)$ & $\begin{array}{c}\text { Final change } \\
(\%)\end{array}$ \\
\hline 1.0 & 6.3 & 1.5 & 1.6 & 0.0 & 0.2 & 0.14 & 65 & 0.0010 & 11 & 100 & 0.1 \\
\hline 1.0 & 6.3 & 1.5 & 1.6 & 0.2 & 0.2 & 0.14 & 65 & 0.0009 & 0 & 60 & 0.0 \\
\hline 1.0 & 6.3 & 1.5 & 1.6 & 0.4 & 0.2 & 0.14 & 65 & 0.0009 & 0 & 20 & 0.0 \\
\hline 1.0 & 6.3 & 1.5 & 1.6 & 0.5 & 0.2 & 0.14 & 65 & 0.0009 & 0 & 0 & 0.0 \\
\hline 1.0 & 6.3 & 1.5 & 1.6 & 0.6 & 0.2 & 0.14 & 65 & 0.0009 & 0 & 20 & 0.0 \\
\hline 1.0 & 6.3 & 1.5 & 1.6 & 0.8 & 0.2 & 0.14 & 65 & 0.0009 & 0 & 60 & 0.0 \\
\hline 1.0 & 6.3 & 1.5 & 1.6 & 1.0 & 0.2 & 0.14 & 65 & 0.0009 & 0 & 100 & 0.0 \\
\hline 1.0 & 6.3 & 1.5 & 1.6 & 1.2 & 0.2 & 0.14 & 65 & 0.0009 & 0 & 140 & 0.0 \\
\hline 1.0 & 6.3 & 1.5 & 1.6 & 1.4 & 0.2 & 0.14 & 65 & 0.0009 & 0 & 180 & 0.0 \\
\hline 1.0 & 6.3 & 1.5 & 1.6 & 1.5 & 0.2 & 0.14 & 65 & 0.0009 & 0 & 200 & 0.0 \\
\hline
\end{tabular}




\begin{tabular}{llll}
\hline $\begin{array}{c}\text { Average } \\
(\%)\end{array}$ & & \\
\hline
\end{tabular}

According to the Table 5 maximum amount of drain discharge change into the initial discharge $(\Delta \mathrm{Q} / \mathrm{Q}) \mathrm{was} 11 \%$ and related to the $100 \%$ decreasing entrance resistance at the drain into the initial $E(\Delta \mathrm{E} / \mathrm{E})$. The minimum of changes was $0 \%$ for other increasing or decreasing of $E$. As average for one percent decrease or increase in $E$, amount of drain discharge is changed $0.0 \%$. This indicated that entrance resistance had minimum of effect on drain discharge into the other drainage parameters.

Table 6 shows obtained results for maximum width of water body in the drain.

Table 6. Obtained results for maximum width of water body in the drain (W)

\begin{tabular}{cccccccccccc}
\hline $\mathrm{Dm}(\mathrm{m})$ & $\begin{array}{c}\mathrm{D} \\
(\mathrm{m})\end{array}$ & $\begin{array}{c}\mathrm{DW} \\
(\mathrm{m})\end{array}$ & $\begin{array}{c}\mathrm{Dd} \\
(\mathrm{m})\end{array}$ & $\begin{array}{c}\mathrm{E} \\
(\mathrm{day} / \mathrm{m})\end{array}$ & $\begin{array}{c}\mathrm{W} \\
(\mathrm{m})\end{array}$ & $\begin{array}{c}\mathrm{K} \\
(\mathrm{m} / \mathrm{day})\end{array}$ & $\begin{array}{c}\mathrm{S} \\
(\mathrm{m})\end{array}$ & $\begin{array}{c}\text { Drain } \\
\text { discharge } \\
(\mathrm{m} / \text { day })\end{array}$ & $\begin{array}{c}\Delta \mathrm{Q} / \mathrm{Q} \\
(\%)\end{array}$ & $\begin{array}{c}\Delta \mathrm{W} / \mathrm{W}(\%) \\
\text { Final } \\
\text { change } \\
(\%)\end{array}$ \\
\hline 1.0 & 6.3 & 1.5 & 1.6 & 0.5 & 0.02 & 0.14 & 65 & 0.0009 & 0 & 90 & 0.0 \\
1.0 & 6.3 & 1.5 & 1.6 & 0.5 & 0.05 & 0.14 & 65 & 0.0009 & 0 & 75 & 0.0 \\
1.0 & 6.3 & 1.5 & 1.6 & 0.5 & 0.10 & 0.14 & 65 & 0.0009 & 0 & 50 & 0.0 \\
1.0 & 6.3 & 1.5 & 1.6 & 0.5 & 0.15 & 0.14 & 65 & 0.0009 & 0 & 25 & 0.0 \\
1.0 & 6.3 & 1.5 & 1.6 & 0.5 & 0.20 & 0.14 & 65 & 0.0009 & 0 & 0 & 0.0 \\
1.0 & 6.3 & 1.5 & 1.6 & 0.5 & 0.25 & 0.14 & 65 & 0.0010 & 11 & 25 & 0.4 \\
1.0 & 6.3 & 1.5 & 1.6 & 0.5 & 0.30 & 0.14 & 65 & 0.0010 & 11 & 50 & 0.2 \\
1.0 & 6.3 & 1.5 & 1.6 & 0.5 & 0.35 & 0.14 & 65 & 0.0010 & 11 & 75 & 0.1 \\
1.0 & 6.3 & 1.5 & 1.6 & 0.5 & 0.40 & 0.14 & 65 & 0.0010 & 11 & 100 & 0.1 \\
1.0 & 6.3 & 1.5 & 1.6 & 0.5 & 0.50 & 0.14 & 65 & 0.0010 & 11 & 150 & 0.1 \\
Average & & & & & & & & & 6 & 71 & 0.1 \\
$(\%)$ & & & & & & & & & 6 & \\
\hline
\end{tabular}

According to the Table 6 maximum amount of drain discharge change into the initial discharge $(\Delta \mathrm{Q} / \mathrm{Q}) \mathrm{was} 11 \%$ and related to the increasing maximum width of water body in the drain into the initial $W(\Delta \mathrm{W} / \mathrm{W})$. The minimum of changes was $0 \%$ for decreasing of $W$. As average for one percent decrease or increase in $W$, amount of drain discharge is changed $0.1 \%$.

Table 7 shows obtained results for hydraulic permeability.

Table 7. Obtained results for hydraulic permeability $(\mathrm{K})$

\begin{tabular}{cccccccccccc}
\hline $\mathrm{Dm}(\mathrm{m})$ & $\begin{array}{c}\mathrm{D} \\
(\mathrm{m})\end{array}$ & $\begin{array}{c}\mathrm{Dw} \\
(\mathrm{m})\end{array}$ & $\begin{array}{c}\mathrm{Dd} \\
(\mathrm{m})\end{array}$ & $\begin{array}{c}\mathrm{E} \\
(\mathrm{day} / \mathrm{m})\end{array}$ & $\begin{array}{c}\mathrm{W} \\
(\mathrm{m})\end{array}$ & $\begin{array}{c}\mathrm{K} \\
(\mathrm{m} / \mathrm{day})\end{array}$ & $\begin{array}{c}\mathrm{S} \\
(\mathrm{m})\end{array}$ & $\begin{array}{c}\text { Drain } \\
\text { discharge } \\
(\mathrm{m} / \text { day })\end{array}$ & $\begin{array}{c}\Delta \mathrm{Q} / \mathrm{Q} \\
(\%)\end{array}$ & $\begin{array}{c}\Delta \mathrm{K} / \mathrm{K}(\%) \\
\text { Final } \\
\text { change } \\
(\%)\end{array}$ \\
\hline 1.0 & 6.3 & 1.5 & 1.6 & 0.5 & 0.2 & 0.01 & 65 & 0.0001 & 89 & 93 & 1.0 \\
1.0 & 6.3 & 1.5 & 1.6 & 0.5 & 0.2 & 0.05 & 65 & 0.0003 & 67 & 64 & 1.0 \\
1.0 & 6.3 & 1.5 & 1.6 & 0.5 & 0.2 & 0.14 & 65 & 0.0009 & 0 & 0 & 0.0 \\
1.0 & 6.3 & 1.5 & 1.6 & 0.5 & 0.2 & 0.20 & 65 & 0.0013 & 44 & 43 & 1.0 \\
1.0 & 6.3 & 1.5 & 1.6 & 0.5 & 0.2 & 0.50 & 65 & 0.0036 & 300 & 257 & 1.2 \\
1.0 & 6.3 & 1.5 & 1.6 & 0.5 & 0.2 & 1.00 & 65 & 0.0065 & 622 & 614 & 1.0 \\
1.0 & 6.3 & 1.5 & 1.6 & 0.5 & 0.2 & 2.00 & 65 & 0.0108 & 1100 & 1329 & 0.8 \\
1.0 & 6.3 & 1.5 & 1.6 & 0.5 & 0.2 & 5.00 & 65 & 0.0170 & 1789 & 3471 & 0.5 \\
1.0 & 6.3 & 1.5 & 1.6 & 0.5 & 0.2 & 7.00 & 65 & 0.0196 & 2078 & 4900 & 0.4 \\
1.0 & 6.3 & 1.5 & 1.6 & 0.5 & 0.2 & 10.00 & 65 & 0.0221 & 2356 & 7043 & 0.3 \\
Average & & & & & & & & & 938 & 1979 & 0.8 \\
$(\%)$ & & & & & & & & & 938 & \\
\hline
\end{tabular}

According to the Table 7 maximum amount of drain discharge change into the initial discharge $(\Delta \mathrm{Q} / \mathrm{Q})$ was $2356 \%$ and related to the $7043 \%$ increasing hydraulic permeability into the initial $K(\Delta \mathrm{K} / \mathrm{K})$. The minimum of changes was $44 \%$ for $43 \%$ increasing of $K$. As average for one percent decrease or increase in $K$, amount of drain discharge is changed $0.8 \%$.

Table 8 shows obtained results for spacing between the parallel drains. 
Table 8. Obtained results for spacing between the parallel drains (S)

\begin{tabular}{|c|c|c|c|c|c|c|c|c|c|c|c|}
\hline $\operatorname{Dm}(\mathrm{m})$ & $\begin{array}{c}\mathrm{D} \\
(\mathrm{m})\end{array}$ & $\begin{array}{l}\text { Dw } \\
(\mathrm{m})\end{array}$ & $\begin{array}{l}\mathrm{Dd} \\
(\mathrm{m})\end{array}$ & $\begin{array}{c}\mathrm{E} \\
(\mathrm{day} / \mathrm{m})\end{array}$ & $\begin{array}{l}\mathrm{W} \\
(\mathrm{m})\end{array}$ & $\begin{array}{c}\mathrm{K} \\
(\mathrm{m} / \text { day })\end{array}$ & $\begin{array}{c}\mathrm{S} \\
(\mathrm{m})\end{array}$ & $\begin{array}{c}\text { Drain discharge } \\
(\mathrm{m} / \text { day })\end{array}$ & $\begin{array}{c}\Delta \mathrm{Q} / \mathrm{Q} \\
(\%)\end{array}$ & $\Delta \mathrm{S} / \mathrm{S}(\%)$ & $\begin{array}{c}\text { Final change } \\
(\%)\end{array}$ \\
\hline 1.0 & 6.3 & 1.5 & 1.6 & 0.5 & 0.2 & 0.14 & 25 & 0.0045 & 400 & 62 & 6.5 \\
\hline 1.0 & 6.3 & 1.5 & 1.6 & 0.5 & 0.2 & 0.14 & 45 & 0.0017 & 89 & 31 & 2.9 \\
\hline 1.0 & 6.3 & 1.5 & 1.6 & 0.5 & 0.2 & 0.14 & 65 & 0.0009 & 0 & 0 & 0.0 \\
\hline 1.0 & 6.3 & 1.5 & 1.6 & 0.5 & 0.2 & 0.14 & 85 & 0.0006 & 33 & 31 & 1.1 \\
\hline 1.0 & 6.3 & 1.5 & 1.6 & 0.5 & 0.2 & 0.14 & 100 & 0.0004 & 56 & 54 & 1.0 \\
\hline 1.0 & 6.3 & 1.5 & 1.6 & 0.5 & 0.2 & 0.14 & 150 & 0.0002 & 78 & 131 & 0.6 \\
\hline 1.0 & 6.3 & 1.5 & 1.6 & 0.5 & 0.2 & 0.14 & 200 & 0.0001 & 89 & 208 & 0.4 \\
\hline 1.0 & 6.3 & 1.5 & 1.6 & 0.5 & 0.2 & 0.14 & 250 & 0.0001 & 89 & 285 & 0.3 \\
\hline 1.0 & 6.3 & 1.5 & 1.6 & 0.5 & 0.2 & 0.14 & 300 & 0.0001 & 89 & 362 & 0.2 \\
\hline 1.0 & 6.3 & 1.5 & 1.6 & 0.5 & 0.2 & 0.14 & 400 & 0.0001 & 89 & 515 & 0.2 \\
\hline $\begin{array}{c}\text { Average } \\
(\%)\end{array}$ & & & & & & & & & 112 & 186 & 1.5 \\
\hline
\end{tabular}

According to the Table 8 maximum amount of drain discharge change into the initial discharge $(\Delta \mathrm{Q} / \mathrm{Q})$ was $400 \%$ and related to the $62 \%$ decreasing drain spacing into the initial $S(\Delta \mathrm{S} / \mathrm{S})$. The minimum of changes was $33 \%$ for $31 \%$ increasing of $S$. As average for one percent decrease or increase in $S$, amount of drain discharge is changed $1.5 \%$.

According to the Tables 1-8, the most amount of change in drain discharge for one percent increase or decrease in each of drainage parameters was owned by depth of water level in drain below soil surface (Dw) equal to $3.0 \%$.

Figure 1 shows trends of drain discharge changes for change of each drainage parameters in subsurface drainage systems.

For increasing $D m$, amount of drain discharge decreased with an almost uniform slope. The amounts of drain discharge increased for $D$ until 20 meters. After this amount, drain discharge remained constant. For increasing of $D w, D d$, and $K$, amount of drain discharge also increased. However, entrance resistance caused drain discharge decrease. The amount of $\mathrm{W}=0.2 \mathrm{~m}$ (initial situation) was an important point because for amount more than it, drain discharge increased. Where amount of $S$ increased from 25 meters to 45 meters, drain discharge decreased with a steep slope and after 150 meters amount of drain discharge remained constant.

Figure 2 shows amounts of minimum, maximum, and average of drain discharge changes for one percent increase or decrease in each of drainage parameters.

In Figure 2 not only the most amount of average changes related to $D w$, but minimum and maximum of drain discharge owned by this parameter. Thus, depth of water level in drain below soil surface is introduced as the most effective parameter between all of the drainage parameters for drain discharge. However, should not be ignored role of drain spacing particularly in low spacings.
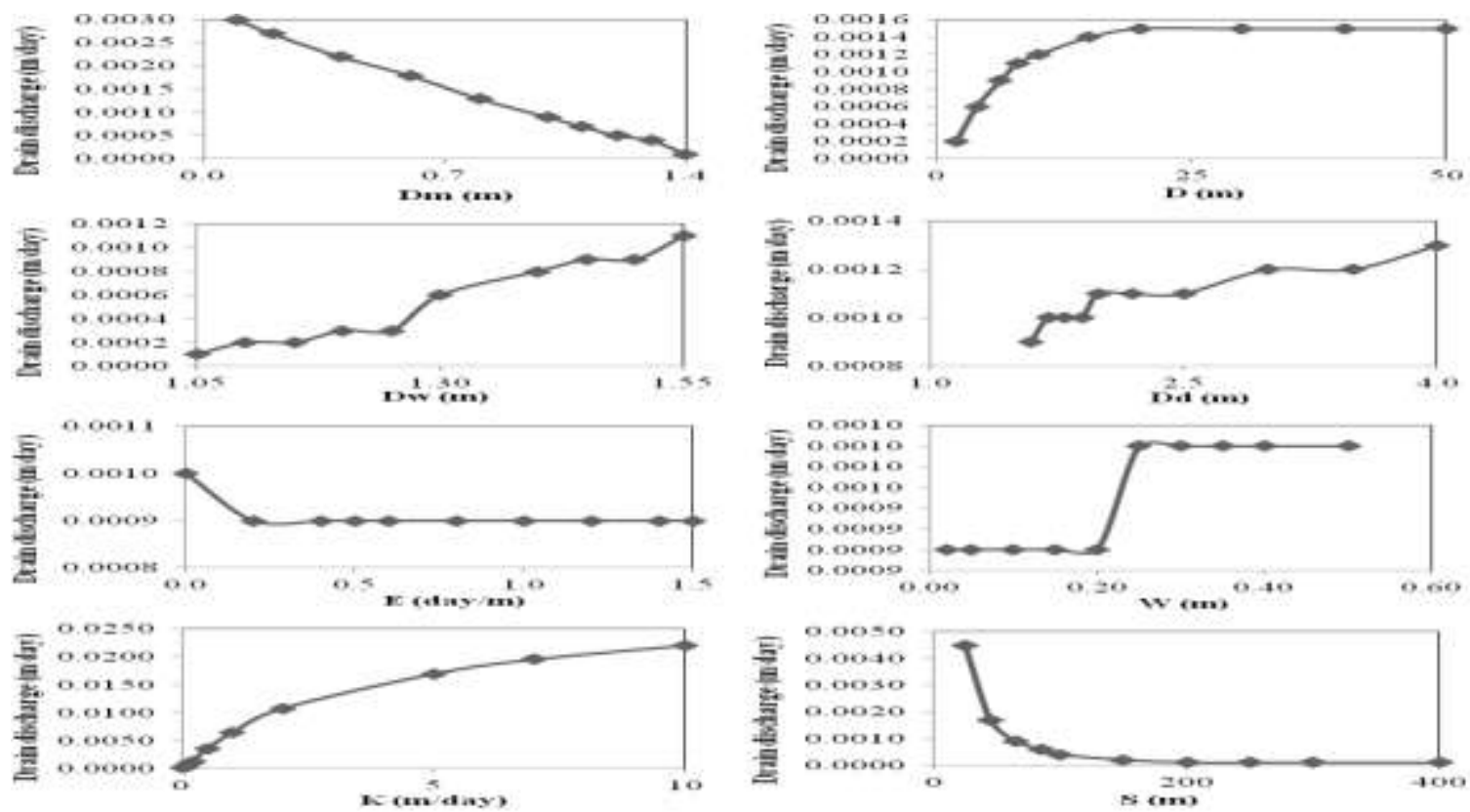

Figure 1. Trends of drain discharge changes for change of each drainage parameters in subsurface drainage systems 


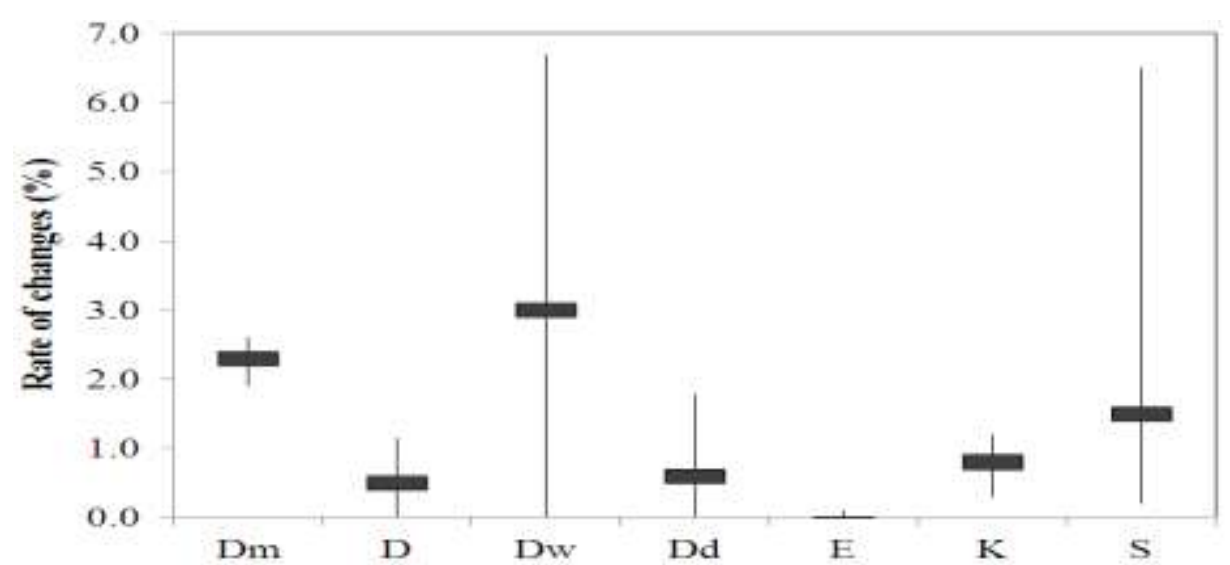

Figure 2. Amounts of minimum, maximum, and average of drain discharge changes for one percent increase or decrease in each of drainage parameters

\section{Conclusion}

Due to the importance of subsurface drainage discharge on the water management, in this paper effect of drainage parameters change on amount of drain discharge investigated in subsurface drainage systems. To summarize, it could be concluded that:

Entrance resistance at the drain had minimum of effect on drain discharge into the other drainage parameters.

The most amount of change in drain discharge for one percent increase or decrease in each of drainage parameters was owned by depth of water level in drain below soil surface equal to $3.0 \%$.

When amount of drain spacing increased from 25 meters to 45 meters, drain discharge decreased with a steep slope and after 150 meters amount of drain discharge remained constant.

Depth of water level in drain below soil surface is introduced as the most effective parameter between all of the drainage parameters for drain discharge. However, should not be ignored role of drain spacing particularly in low spacings.

\section{References}

[1] Ahmadi M.Z. 1995. A field approach to estimation of humid area drainage coefficients, Agricultural Water Management, 29 (1), pp. 101-109. DOI: http://dx.doi.org/10.1016/0378-3774(95)01186-2

[2] Ali M.H. 2011. Drainage of Agricultural Lands, Practices of Irrigation \& On-farm Water Management: Volume 2, pp. 327-378. DOI: $10.1007 / 978-1-4419-7637-6 \_9$

[3] Barua G. and K.N. Tiwari, 1995. Theories of seepage into auger holes in homogeneous anisotropic soil, Journal of Hydrology, 167 (1-4), pp. 1-22. DOI: http://dx.doi.org/10.1016/0022-1694(94)02629-P

[4] Basu D. and M. Prezzi 2010. Design Charts for Vertical Drains Considering Soil Disturbance, Geotechnical Modeling, pp. $420-429$. DOI: http://dx.doi.org/10.1061/41095(365)39

[5] Brandyk T., P.B. Leeds-Harrison and K. Skapski 1992. A simple flow resistance model for the management of drainage/subirrigation systems, Agricultural Water Management, 21 (1-2), pp. 67-77. DOI: http://dx.doi.org/10.1016/0378-3774(92)90083-9

[6] Burdon D.J. 1986. Hydrogeological aspects of agricultural drainage in Ireland, Environmental Geology and Water Sciences, 9 (1), pp. 41-65 DOI: 10.1007/BF02439885

[7] Castanheira P.J. and F.L. Santos 2009. A simple numerical analyses software for predicting water table height in subsurface drainage, Irrigation and Drainage Systems, 23 (4), pp. 153-162. DOI: 10.1007/s10795-009-9079-5

[8] Choudhry M.R., A. Khaliq, W.F. Vlotman, H.U. Rehman 1995. Physical and hydraulic properties of synthetic envelopes for subsurface drainage in Pakistan, Irrigation and Drainage Systems, 9 (1), pp. 73-84. DOI: 10.1007/BF00881589

[9] Coles E.D. 1968. SOME NOTES ON DRAINAGE DESIGN PROCEDURE. Proceedings of The South African Sugar Technologists' Association, pp. 189-199.

[10] Cooke R.A., S. Badiger and A .M Garcia 2001. Drainage equations for random and irregular tile drainage systems, Agricultural Water Management, 48 (3), pp. 207-224. DOI: http://dx.doi.org/10.1016/S0378-3774(00)00136-0

[11] Christen E.W., J.E. Ayars and J.W. Hornbuckle 2001. Subsurface drainage design and management in irrigated areas of Australia, Irrigation Science, 21 (1). pp. 35-43. DOI: 10.1007/s002710100048

[12] Endres A.L., J.P. Jones and E.A. Bertrand 2006. Pumping-induced vadose zone drainage and storage in an unconfined aquifer: A comparison of analytical model predictions and field measurements, Journal of Hydrology, 335 (1-2), pp. 207-218. DOI: http://dx.doi.org/10.1016/j.jhydrol.2006.07.018

[13] French B.E. and J.R. O'Callaghan 1966. A field test of drain spacing equations for agricultural land, Journal of Agricultural Engineering Research, 11(4), pp. 282-295. DOI: http://dx.doi.org/10.1016/S0021-8634(66)80036-7

[14] Gallichand J. 1994. Numerical simulations of steady-state subsurface drainage with vertically decreasing hydraulic conductivity, Irrigation and Drainage Systems, 8 (1), pp. 1-12.

[15] Geng X., B. Indraratna and C. Rujikiatkamjorn 2012. Analytical Solutions for a Single Vertical Drain with Vacuum and TimeDependent Surcharge Preloading in Membrane and Membraneless Systems, International Journal of Geomechanics, 11 (1), pp. 27 42. DOI: http://dx.doi.org/10.1061/(ASCE)GM.1943-5622.0000106

[16] Ghandeharioon A., B. Indraratna and C. Rujikiatkamjorn 2010. Analysis of Soil Disturbance Associated with Mandrel-Driven Prefabricated Vertical Drains Using an Elliptical Cavity Expansion Theory, International Journal of Geomechanics, 10 (2), pp. 5364. DOI: http://dx.doi.org/10.1061/(ASCE)GM.1943-5622.0000027 
[17] Gureghian A.B. and E.G. Youngs 1975. The calculation of steady-state water-table heights in drained soils by means of the finiteelement method, Journal of Hydrology, 27 (1-2), pp. 15-32. DOI: http://dx.doi.org/10.1016/0022-1694(75)90096-7

[18] Hanson B.R. and J.E. Ayars 2002. Strategies for reducing subsurface drainage in irrigated agriculture through improved irrigation, Irrigation and Drainage Systems, 16 (4), pp. 261-277. DOI: 10.1023/A:1024861112978

[19] Hirekhan M., S.K. Gupta and K.L. Mishra 2007. Application of WaSim to assess performance of a subsurface drainage system under semi-arid monsoon climate, Agricultural Water Management, 88 (1-3), pp. 224-234. DOI: http://dx.doi.org/10.1016/j.agwat.2006.12.001

[20] Hornbuckle J.W., E.W. Christen, J.E. Ayars and R.D. Faulkner 2005. Controlled water table management as a strategy for reducing salt loads from subsurface drainage under perennial agriculture in semi-arid Australia, Irrigation and Drainage Systems, 19 (2), pp. 145-159. DOI: 10.1007/s10795-005-4420-0

[21] Howell R., E.M. Rathje, R. Kamai and R. Boulanger 2012. Centrifuge Modeling of Prefabricated Vertical Drains for Liquefaction Remediation, Journal of Geotechnical and Geoenvironmental Engineering, 137 (3), pp. $262-271 . \quad$ DOI: http://dx.doi.org/10.1061/(ASCE)GT.1943-5606.0000604

[22] Hunt B. 2005. Flow to Vertical and Nonvertical Wells in Leaky Aquifers, Journal of Hydrologic Engineering, 10 (6), pp. $477-484$. DOI: http://dx.doi.org/10.1061/(ASCE)1084-0699(2005)10:6(477)

[23] Kannan N. 2008. Study of Drawdown-Drain Discharge Relationship and its Application in Design of Cost Effective Subsurface Drainage System in Mugogo Swamp, Busogo, Rwanda, Water Resources Management, 22 (8), pp. 1113-1125. DOI: $10.1007 / \mathrm{s} 11269-007-9215-7$

[24] Lovell C.J. and E.G. Youngs 1984. A comparison of steady-state land-drainage equations, Agricultural Water Management, 9 (1), pp. 1-21. DOI: http://dx.doi.org/10.1016/0378-3774(84)90015-5

[25] Luan M. and Y. Leng 2008. A Comparative Study on Monotonic Shear Behaviors of Granular Soils Under Different Drainage Conditions, Geotechnical Engineering for Disaster Mitigation and Rehabilitation, pp. 1068-1078. DOI: 10.1007/978-3-540-79846$0 \_141$

[26] Marinucci A., E.M. Rathje, J.S. Ellington, B.R. Cox, F.Y. Menq and K.H. Stokoe 2010. Evaluation of the Effectiveness of Prefabricated Vertical Drains Using Full-Scale In Situ Staged Dynamic Testing, Art of Foundation Engineering Practice, pp. 380394. DOI: http://dx.doi.org/10.1061/41093(372)17

[27] Molen W.H.V.D. and J. Wesseling 1991. A solution in closed form and a series solution to replace the tables for the thickness of the equivalent layer in Hooghoudt's drain spacing formula, Agricultural Water Management, 19 (1), pp. 1-16. DOI: http://dx.doi.org/10.1016/0378-3774(91)90058-Q

[28] Moustafa M.M. 1998. Time-dependent drainage from root zone and drainage coefficient under different irrigation management levels for subsurface drainage design in Egypt, Irrigation and Drainage Systems, 12 (2), pp. 141-159. DOI: 10.1023/A:1006055704143

[29] Nwa E.U. and J.G. Twocock 1969. Drainage design theory and practice, Journal of Hydrology, 9 (3), pp. 259-276. DOI: http://dx.doi.org/10.1016/0022-1694(69)90021-3

[30] O’Kelly B.C. 2006. Compression and consolidation anisotropy of some soft soils, Geotechnical \& Geological Engineering, 24 (6), pp. 1715-1728. DOI: 10.1007/s10706-005-5760-0

[31] O'Neill H.J., T.L. Pollock, H.S. Bailey, P. Milburn, C. Gartley and J.E. Richards 1989. Dinoseb presence in agricultural subsurface drainage from potato fields in northwestern New Brunswick, Canada, Bulletin of Environmental Contamination and Toxicology, 43 (6), pp. 935-940. DOI: 10.1007/BF01702068

[32] Oosterbaan R.J. 1988. Agricultural criteria for subsurface drainage: A systems analysis, Agricultural Water Management, 14 (1-4), pp. 79-90. DOI: http://dx.doi.org/10.1016/0378-3774(88)90063-7

[33] Oosterbaan R.J. 1988. Effectiveness and Social/Environmental Impacts of Irrigation Projects: a Criticial Review., In: ILRI Annual Report 1988, p.18-34, International Institute for Land Reclamation and Improvement, Wageningen, The Netherlands. This paper was presented at the 3rd National Irrigation and Drainage Symposium, 20 to 23 September 1988, Izmir, Turkey.

[34] Oosterbaan R.J. 1991. Agricultural Land Drainage: a wider application through caution and restraint., Lecture delivered at the symposium held to mark the occasion of the 30th International Course on Land Drainage, 27 November 1991. Published in: ILRI Annual Report 1991, p.21-36, International Institute for Land Reclamation and Improvement, Wageningen, The Netherlands.

[35] Oosterbaan R.J. 2010. Spate Irrigation: Water Harvesting and Agricultural Land Development Options in the NWFR of Pakistan, Paper submitted to the International Policy Workshop "Water Management and Land Rehabilitation, NW Frontier Region, Pakistan", Islamabad, December 6 - 8, 2010

[36] Osiensky J.L., R.E. Williams, B. Williams, G. Johnson 2000. Evaluation of drawdown curves derived from multiple well aquifer tests in heterogeneous environments, Mine Water and the Environment, 19 (1), pp 30-55. DOI: 10.1007/BF02687263

[37] Prasad K.S.H., C.S.P. Ojha, P.N. Chandramouli and C.A. Madramootoo 2010. Estimation of Unsaturated Hydraulic Parameters from Infiltration and Internal Drainage Experiments, Journal of Irrigation and Drainage Engineering, 136 (11), pp. 766-773. DOI: http://dx.doi.org/10.1061/(ASCE)IR.1943-4774.0000234

[38] Prasher S.O., A. Madani, R.S. Clemente, G.Q. Geng and A. Bhardwaj 1996. Evaluation of two water table management models for Atlantic Canada, Agricultural Water Management, 32 (1), pp. 49-69. DOI: http://dx.doi.org/10.1016/S0378-3774(96)01260-7

[39] Prasher S.O., S.F. Barrington and A.M. Darbary 1994. Economical design of water table management systems in humid areas, Computers and Electronics in Agriculture, 10 (3), pp. 229-244. DOI: http://dx.doi.org/10.1016/0168-1699(94)90043-4

[40] Rimidis A. and W. Dierickx 2003. Evaluation of subsurface drainage performance in Lithuania, Agricultural Water Management, 59 (1), pp. 15-31. DOI: http://dx.doi.org/10.1016/S0378-3774(02)00111-7

[41] Samani N., M. Kompani-Zare, H. Seyyedian and D.A. Barry 2004. Flow to horizontal and slanted drains in anisotropic unconfined aquifers, Developments in Water Science, 55 (1), pp. 427-440. DOI: http://dx.doi.org/10.1016/S0167-5648(04)80070-4

[42] Singh B. and J.R. O'Callaghan 1978. Non-steady drainage in a layered soil, Journal of Agricultural Engineering Research, 23 (4), pp. 417-427. DOI: http://dx.doi.org/10.1016/0021-8634(78)90056-2

[43] Singh K.M., O.P. Singh, S. Ram and H.S. Chauhan 1992. Modified steady state drainage equations for transient conditions in subsurface drainage, Agricultural Water Management, 20 (4), pp. 329-339. DOI: http://dx.doi.org/10.1016/0378-3774(92)90006-I

[44] Singh M.P., H.S. Chauhan and S. Ram 1996. Unsteady state drainage in a vertically heterogeneous soil, Agricultural Water Management, 31 (3), pp. 285-293. DOI: http://dx.doi.org/10.1016/0378-3774(96)01239-5

[45] Singh P.K., O.P. Singh, C.S. Jaiswal and H.S. Chauhan 1999. Subsurface drainage of a three layered soil with slowly permeable top layer, Agricultural Water Management, 42 (1), pp. 97-109. DOI: http://dx.doi.org/10.1016/S0378-3774(99)00027-X

[46] Singh S.K. 2010. Generalized Analytical Solutions for Groundwater Head in Inclined Aquifers in the Presence of Subsurface Drains, Journal of Irrigation and Drainage Engineering, 136 (3), pp. 194-203. DOI: http://dx.doi.org/10.1061/(ASCE)IR.19434774.0000150 
[47] Skaggs R.W., M.A. Youssef and G.M. Chescheir 2006. Drainage design coefficients for eastern United States, Agricultural Water Management, 86 (1-2), pp. 40-49. DOI: http://dx.doi.org/10.1016/j.agwat.2006.06.007

[48] Valipour M. 2012. A Comparison between Horizontal and Vertical Drainage Systems (Include Pipe Drainage, Open Ditch Drainage, and Pumped Wells) in Anisotropic Soils, American-Eurasian Journal of Agricultural \& Environmental Sciences.

[49] Wahba M.A.S. and E.W. Christen 2006. Modeling subsurface drainage for salt load management in southeastern Australia, Irrigation and Drainage Systems, 20 (2-3), pp. 267-282. DOI: 10.1007/s10795-006-9007-x

[50] Wesseling J. 1964. A comparison of the steady state drain spacing formulas of Hooghoudt and Kirkham in connection with design practice, Journal of Hydrology, 2 (1), pp. 25-32. DOI: http://dx.doi.org/10.1016/0022-1694(64)90062-9

[51] Wesseling J. 1964. The effect of using continually submerged drains on drain spacings, Journal of Hydrology, 2 (1), pp. $33-43$. DOI: http://dx.doi.org/10.1016/0022-1694(64)90063-0

[52] Wiskow E. and R.R.V.D. Ploeg 2003. Calculation of drain spacings for optimal rainstorm flood control, Journal of Hydrology, 272 (1-4), pp. 163-174. DOI: http://dx.doi.org/10.1016/S0022-1694(02)00262-7

[53] Youngs E.G. 1976. Determination of the variation of hydraulic conductivity with depth in drained lands and the design of drainage installations, Agricultural Water Management, 1 (1), pp. 57-66. DOI: http://dx.doi.org/10.1016/0378-3774(76)90007-X

[54] Youngs E.G. 1985. A simple drainage equation for predicting water-table drawdowns, Journal of Agricultural Engineering Research, 31 (4), pp. 321-328. DOI: http://dx.doi.org/10.1016/0021-8634(85)90108-8

[55] Youngs E.G. 1986. Water-table heights in drained anisotropic homogeneous soils, Agricultural Water Management, 11 (1), pp. 111. DOI: http://dx.doi.org/10.1016/0378-3774(86)90031-4

[56] Youngs E.G. 1991. A note on the power-law land-drainage equation for deep soils, Journal of Agricultural Engineering Research, 49, pp. 161-163. DOI: http://dx.doi.org/10.1016/0021-8634(91)80036-E 